01

\title{
Внутридопплеровские резонансы затухания свободной поляризации в ультратонких газовых ячейках
}

\author{
(C) А.Ч. Измайлов \\ Институт физики Национальной академии наук Азербайджана, \\ AZ-1143 Баку, Азербайджан \\ e-mail: azizm57@rambler.ru
}

Поступила в редакцию 21.09.2020 г.

В окончательной редакции 21.09.2020 г.

Принята к публикации 08.10.2020 г.

\begin{abstract}
Теоретически исследован линейно-оптический эффект затухания свободной поляризации (ЗСП) в ультратонкой газовой ячейке, внутренняя толщина которой меньше или порядка длины волны возбуждающего монохроматического лазерного излучения, пропускаемого ортогонально плоскопараллельным стенкам данной ячейки. Рассматриваемый когерентный сигнал ЗСП испускается сразу после резкого прерывания такого сравнительно слабого стационарного излучения. Установлены и проанализированы внутридопплеровские резонансы, возникающие на центральных частотах исследуемых квантовых переходов в спектрах интенсивности и энергии ЗСП вследствие пролетной релаксации атомов в газовой ячейке. Показана существенная зависимость данных резонансов и динамики ЗСП от отношения внутренней толщины ультратонкой ячейки к длине волны возбуждающего монохроматического излучения. Обнаруженные и исследованные в линейно-оптическом режиме узкие, высококонтрастные внутридопплеровские резонансы ЗСП могут быть использованы в атомной (молекулярной) спектроскопии сверхвысокого разрешения, а также в качестве реперов для компактных стандартов частоты.
\end{abstract}

Ключевые слова: внутридопплеровские резонансы, затухание свободной поляризации, ультратонкая газовая ячейка, когерентное излучение, фазы дипольных моментов.

DOI: $10.21883 /$ OS.2021.01.50435.238-20

\section{Введение}

В настоящее время создаются и используются в спектроскопии высокого разрешения ультратонкие газовые ячейки, внутренняя толщина которых (между их плоскопараллельными стенками) меньше или порядка длины волны $\lambda \sim 1 \mu \mathrm{m}$ облучающего света [1]. При зондировании атомов в таких ячейках монохроматическим лазерным излучением в линейно-оптическом режиме на частотах квантовых переходов регистрируются узкие внутридопплеровские резонансы поглощения, обусловленные эффектом Дике [2]. Структура данных резонансов существенно зависит от отношения внутренней толщины ячейки $l$ к длине волны $\lambda \sim 1 \mu \mathrm{m}$ облучающего света [2-4]. Эти резонансы, в частности, используются для прецизионного спектрального анализа взаимодействия атомов с поверхностью [5]. Кроме того, такие узкие резонансы могут быть эффективными реперами для высокоточных стандартов частоты вследствие пренебрежимо слабой зависимости светового сдвига и уширения данных линейно-оптических резонансов от интенсивности индуцирующего лазерного излучения. Однако слабость сигнала светового поглощения в подобных ультратонких ячейках с разреженной газовой средой существенно ограничивает возможности использования упомянутых внутридопплеровских резонансов.

В связи с этим может представить интерес исследование сигнала затухания свободной поляризации
(3СП) [6], который обусловлен когерентным излучением, испускаемым атомами (молекулами) газовой среды после резкого прерывания исходного возбуждающего лазерного излучения в некоторый момент времени $t=t_{0}$ (рис. 1). Действительно, излучение накачки с частотой, близкой к центру спектральной линии некоторого атомного перехода $a \leftrightarrow b$, создает суперпозицию квантовых уровней $a$ и $b$. В результате в среде образуется совокупность осциллирующих дипольных моментов. После прерывания исходного лазерного излучения такие атомные диполи продолжают некоторое время колебаться и испускать регистрируемый сигнал ЗСП [6]. Данный когерентный сигнал распространяется в направлении возбуждающего света, поскольку только в этом направлении излучение этих диполей оказывается сфазированным. Вследствие поступательного движения атомов

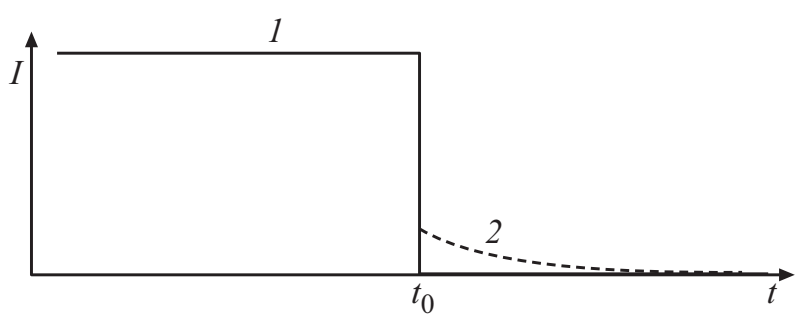

Рис. 1. Интенсивность $I$ возбуждающего излучения (1), прерываемого в момент времени $t=t_{0}$, и последующий испускаемый сигнал ЗСП (2). 
(молекул) газовой среды происходит рассогласование по фазе их дипольных моментов, что и является причиной наблюдаемого сигнала ЗСП [6].

В работе автора [7] были теоретически исследованы особенности ЗСП в ультратонкой газовой ячейке после воздействия ультракороткого интенсивного импульса лазерного излучения. Так был установлен новый механизм ЗСП, обусловленный спецификой рассогласования фаз светоиндуцированных атомных дипольных моментов вследствие пролетной релаксации атомов в такой ячейке. В результате динамика ЗСП, установленная в работе [7], радикально отличается от известного случая [6] в обычной (макроскопической) газовой ячейки.

В отличие от упомянутой работы [7] в настоящей работе теоретически исследовано ЗСП, возникающее в линейно-оптическом режиме в ультратонкой газовой ячейке после резкого прерывания достаточно слабого стационарного монохроматического лазерного пучка (рис. 1). Установлено, что в спектральных характеристиках интенсивности и энергии такого сигнала ЗСП на центральных частотах светоиндуцированных квантовых переходов возникают внутридопплеровские резонансы, структура которых существенно зависит от отношения внутренней толщины ячейки $l$ к длине волны $\lambda$ возбуждающего излучения. Анализируются условия, при которых данные резонансы ЗСП могут найти применение в атомной (молекулярной) спектроскопии сверхвысокого разрешения, а также в качестве реперов для компактных стандартов частоты и времени.

\section{Основные соотношения}

Рассмотрим разреженную газовую среду между плоскопараллельными прозрачными стенками одномерной ячейки с внутренней толщиной $l$. Этот газ полагается настолько разреженным, что взаимодействием между его атомами (молекулами) можно пренебречь. Ортогонально стенкам ячейки вдоль оси $z$ через газовую среду распространяется стационарное монохроматическое излучение, которое резко прерывается в момент времени $t_{0}=0$ (рис. 1). Электрическая компонента данного излучения имеет вид

$$
\mathscr{E}(z, t)=0.5 \mathbf{e} E \eta(-t) \exp [i(\omega t-k z)]+c . c .,
$$

где $E$ и е - соответственно амплитуда и единичный вектор поляризации излучения, $\omega-$ его частота, $k=2 \pi / \lambda-$ волновое число, $\lambda-$ длина волны, $c . c .-$ комплексно-сопряженная величина, $\eta(x)$ - ступенчатая функция $(\eta(x)=1$, если $x \geq 0$, и $\eta(x)=0$, если $x<0)$. Частота излучения $\omega$ близка к центральной частоте $\omega_{0}$ квантового перехода $a \leftrightarrow b$ между основным $a$ и возбужденным $b$ атомными уровнями. Интенсивность излучения (1) полагается настолько низкой, что его взаимодействие с атомами газа происходит в линейнооптическом режиме. Тогда можно написать следующее уравнение для элемента атомной матрицы плотности оптической когерентности $\rho_{a b}[8]$ :

$$
\begin{aligned}
\frac{\partial \rho_{a b}}{\partial t} & +v \frac{\partial \rho_{a b}}{\partial z}= \\
& =0.5 i \Delta N \frac{E(\mathbf{e d})}{\hbar} \eta(-t) \exp [i(\delta t-k z)]-\gamma \rho_{a b}
\end{aligned}
$$

где $\delta=\left(\omega-\omega_{0}\right)$ - отстройка частоты $\omega$ излучения (1) от резонанса с переходом $a \leftrightarrow b$ с естественной полушириной $\gamma$ спектральной линии и матричным элементом d дипольного момента, $v$ - проекция атомной скорости на ось $z, \Delta N=\left(N_{b}-N_{a}\right)$ - разность равновесных населенностей $N_{b}$ и $N_{a}$ уровней $b$ и $a$. Величина $\rho_{a b}$ должна удовлетворять также граничным условиям, которые зависят от особенностей столкновений атомов (молекул) газа со стенками рассматриваемой ячейки, расположенных в плоскостях с координатами $z=0$ и l. Как и в предыдущих теоретических исследованиях для тонких газовых ячеек $[3,4,7]$, будем считать, что в результате каждого из таких столкновений происходит полное разрушение оптической когерентности.

Произведем следующую замену в соотношении (2):

$$
\rho_{a b}=\sigma_{a b} \exp [i(\delta t-k z)]
$$

Тогда из (2) вытекает уравнение для величины $\sigma_{a b}$ :

$$
\begin{aligned}
\frac{\partial \sigma_{a b}}{\partial t} & +v \frac{\partial \sigma_{a b}}{\partial z}+[\gamma+i(\delta-k v)] \sigma_{a b}= \\
& =0.5 i \Delta N \frac{E(\mathbf{e d})}{\hbar} \eta(-t) .
\end{aligned}
$$

С учетом отмеченных граничных условий находим следуюшее решение $\sigma_{a b}$ уравнения (4) для времени $t \geq 0$, когда проявляется сигнал ЗСП:

$$
\begin{gathered}
\sigma_{a b}(z, v, t)=0.5 i \Delta N \frac{E(\mathbf{e d})}{\hbar \Lambda} \eta(t)\{[\exp (-\Lambda t) \\
\left.-\exp \left(-\Lambda \frac{z}{|v|}\right)\right] \eta(z-v t) \eta(v)+[\exp (-\Lambda t) \\
\left.\left.-\exp \left(-\Lambda \frac{(l-z)}{|v|}\right)\right] \eta(l-z+v t) \eta(-v)\right\},
\end{gathered}
$$

где

$$
\Lambda=\gamma+i(\delta-k v) .
$$

Заметим, что в зависящей от времени функции $\exp (-\Lambda t)$ в соотношении (5) множитель $\exp (-\gamma t)$ описывает радиационный распад оптической когерентности, а множитель $\exp (i k v t)$ определяется изменением фазы $k v t$ соответствующих атомных диполей с проекцией скорости $v[6]$.

Излучение (1) индуцирует поляризацию газовой среды следующего вида $[6,8]$ :

$$
\mathbf{Q}(z, t)=0.5 \mathbf{e} P(z, t) \exp [i(\omega t-k z)]+c . c .,
$$


где

$$
P(z, t)=2 d \int_{-\infty}^{\infty} \sigma_{a b}(z, v, t) F(v) d v
$$

величина $\sigma_{a b}$ определяется формулами (3) и $(5), F(v)-$ распределение Максвелла по проекции атомной скорости $v$ на ось $z$ :

$$
F(v)=\pi^{-0.5} u^{-1} \exp \left(-v^{2} u^{-2}\right),
$$

где $u$ - наиболее вероятная скорость атомов в газе.

Несмотря на прерывание возбуждающего излучения (1) в момент времени $t_{0}=0$ (рис. 1), индуцируемая им поляризация среды (7), (8) является источником когерентного сигнала ЗСП при $t>0$, электрическая компонента которого имеет вид [6]

$$
\mathscr{E}_{S}(z, t)=0.5 \mathbf{e} E_{S}(z, t) \exp [i(\omega t-k z)]+c . c . .
$$

На основе уравнений Максвелла [6,8] устанавливается связь амплитуды $E_{s}$ сигнала ЗСП $(10)$ на выходе из ячейки с соответствующей компонентой поляризации среды (7), (8):

$$
\begin{aligned}
E_{S}(l, t)= & -2 i \pi k \int_{0}^{l} P(z, t) d z=-4 i \pi k d \int_{-\infty}^{\infty} F(v) \\
& \times\left[\int_{0}^{l} \sigma_{a b}(z, v, t) d z\right] d v
\end{aligned}
$$

где величина $\sigma_{a b}$ определяется формулой (5) и ее интегральное значение по внутренней толщине ячейки $l$ в (11) имеет вид

$$
\begin{aligned}
& \int_{0}^{l} \sigma_{a b}(z, v, t) d z=0.5 i \Delta N \frac{E(\mathbf{e d})}{\hbar \Lambda} \eta(t) \eta(l-|v| t) \\
& \times\left\{\exp (-\Lambda t)(l-|v| t)-\frac{|v|}{\Lambda}\left[\exp (-\Lambda t)-\exp \left(-\Lambda \frac{l}{|v|}\right)\right]\right\} .
\end{aligned}
$$

Наряду с интенсивностью $I=\left|E_{S}(l, t)\right|^{2}$ сигнала ЗСП (11) на эксперименте может быть измерена также следующая энергетическая характеристика $R(l, \Delta)$ такого сигнала за время его регистрации, начиная с некоторого момента $t=\Delta>0$ :

$$
R(l, \Delta)=\int_{\Delta}^{\infty}\left|E_{s}(l, t)\right|^{2} d t .
$$

\section{Обсуждение результатов}

На рис. 2 представлены численно рассчитанные зависимости интенсивности $I=\left|E_{s}(l, t)\right|^{2}$ сигнала ЗСП (11) от расстройки частоты $\delta$ возбуждающего излучения (1)

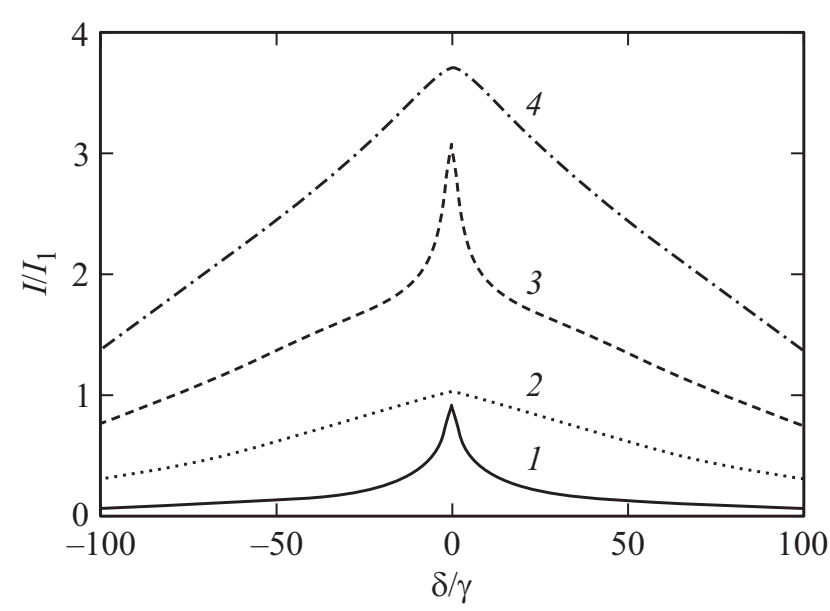

Рис. 2. Зависимость интенсивности $I$ сигнала ЗСП от расстройки частоты $\delta$ (в единицах $\gamma$ ) в начальный момент времени $t=t_{0}=0$ при $l / \lambda=0.5(1), 1(2), 1.5$ (3) и 2 (4), когда $k u=10^{2} \gamma . I_{1}-$ значение интенсивности $I$ при $l=\lambda, \delta=0$ и $t=0$.

в момент времени $t=t_{0}=0$ его прерывания (рис. 1) при различных значениях внутренней толщины $l$ ультратонкой ячейки для спектральной линии резонансного перехода $a \leftrightarrow b$ с допплеровским уширением $k u=10^{2} \gamma$. Данные зависимости подобны известным теоретически рассчитанным спектрам коэффициента поглощения стационарного бегущего монохроматического лазерного пучка в линейно-оптическом режиме $[3,4]$. Действительно, структура этих зависимостей на рис. 2 также в значительной степени определяется отношением $l / \lambda$ внутренней толщины ячейки $l$ к длине волны $\lambda$ лазерного излучения. В частности, при полуцелых отношениях $l / \lambda=0.5$ и 1.5 в спектре интенсивности ЗСП возникает узкий высококонтрастный внутридопплеровский резонанс с центром в точке $\delta=0$ (кривые 1 и 3 на рис. 2). Однако такие резонансы фактически отсутствуют при целых значениях $l / \lambda$ (кривые 2 и 4 на рис. 2). Согласно проведенным расчетам, с ростом толщины ячейки в области $l>2 \lambda$ качественные отличия между рассматриваемыми спектральными зависимостями при различных значениях $l / \lambda$ уменьшаются вплоть до полного исчезновения их внутридопплеровской структуры, когда $l \gg \lambda$.

На рис. 3 показаны зависимости интенсивности $I=\left|E_{s}(l, t)\right|^{2}$ сигнала ЗСП (11) от времени $t>0$ при нулевой расстройке частоты $\delta=0$ для различных значений толщины ячейки $l$. Данные зависимости также в значительной степени определяются отношением $l / \lambda$. Так, при полуцелых значениях $l / \lambda=0.5$ и 1.5 наблюдается гораздо более длительный сигнал ЗСП по сравнению с целыми значениями $l / \lambda=1$ и 2 (рис. 3). Действительно, рассогласование фаз излучения атомных диполей через время $t>0$ после прекращения действия возбуждающего излучения (1) описывается величиной $k v t$ для атомов с различными проекциями скорости $v$ [6-8]. Вследствие столкновительной релаксации на плоскопараллельных 


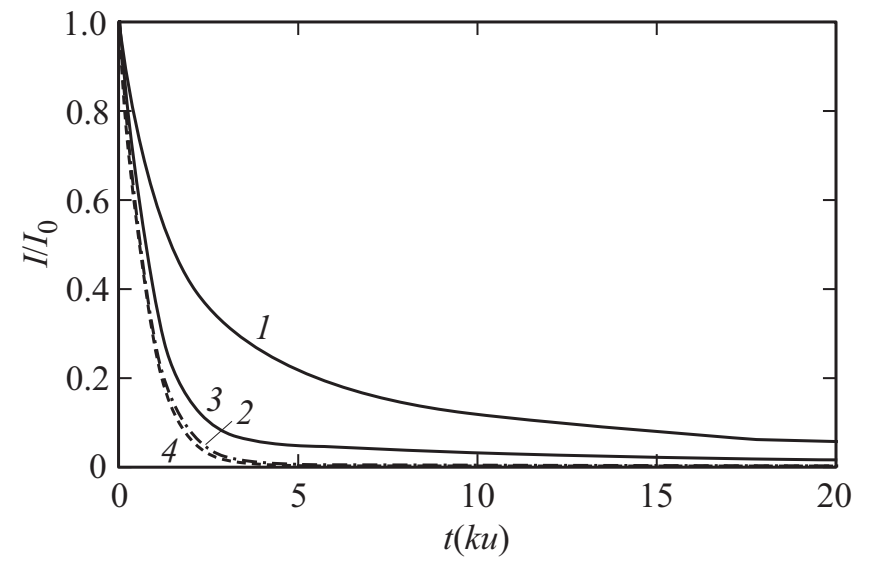

Рис. 3. Зависимость интенсивности $I$ сигнала ЗСП от времени $t>0$ (в единицах $k^{-1} u^{-1}$ ) при нулевой расстройке частоты $\delta=0$ для $l / \lambda=0.5(1), 1(2), 1.5$ (3) и $2(4)$, когда $k u=10^{2} \gamma$. $I_{0}$ - значение интенсивности $I$ при $\delta=0$ в момент времени $t=t_{0}=0$.

стенках ультратонкой ячейки через время $t>0$ оптическая когерентность сохраняется только у атомов газа с проекциями скорости $|v| \leq l / t$, для которых разброс фаз $k|v| t \leq k l=2 \pi(l / \lambda)$. Если такой разброс $k l$ кратен $2 \pi$, то при расстройке лазерной частоты $\delta=0$ вклады в сигнал ЗСП атомных диполей с фазами излучения, сдвинутыми на $\pi$, будут равны по величине и противоположны по знаку. Таким образом, при целых отношениях $l / \lambda$ происходит сравнительно быстрое ЗСП вследствие полного рассогласования фаз всей совокупности атомных диполей в газовой ячейке (кривые 2 и 4 на рис. 3). При других значениях $k l=2 \pi(l / \lambda)$ вклад в ЗСП диполей с некоторыми фазами излучения не будет скомпенсирован противофазными диполями. Особенно ярко это проявляется при полуцелых значениях $l / \lambda=0.5$ и 1.5 , когда сигнал ЗСП наиболее продолжителен (кривые 1 и 3 на рис. 3). Согласно проведенным расчетам, при толщине ячейки $l \gg \lambda$ характерное время ЗСП определяется малой величиной порядка $3(k u)^{-1}$ (как для кривой 4 на рис. 3) и практически не зависит от отношения $l / \lambda$.

На рис. 4 представлены зависимости интенсивности $I=\left|E_{s}(l, t)\right|^{2}$ сигнала ЗСП (11) от расстройки частоты $\delta$ возбуждающего излучения в различные моменты времени $t \geq 0$ после прерывания действия возбуждающего излучения для двух значений толщины ячейки $l=0.5 \lambda$ и $1.5 \lambda$, когда, согласно рис. 2 , проявляется наиболее узкий, высококонтрастный внутридопплеровский резонанс с центром в точке $\delta=0$. Видно, что с течением времени $(t>0)$ имеет место уменьшение не только амплитуды, но и ширины соответствующих зависимостей на рис. 4. Действительно, в рассматриваемом случае неоднородно уширенной спектральной линии резонансного перехода $a \leftrightarrow b$ возбуждающее монохроматическое излучение (1) эффективно взаимодействует лишь с атомами газа, проекции скорости которых $v$ близки к значению $\delta / k$, когда $|\delta-k v| \leq \gamma$ [8]. Вследствие пролетной релаксации в ячейке через время $t>0$ оптическая когерентность сохранится только у атомов с проекциями скорости $|v| \leq l / t$, которым соответствуют расстройки частоты $|\delta| \leq k l / t$. Поскольку именно данные атомы формируют сигнал ЗСП, то зависимость его интенсиности от $\delta$ сужается со временем $t>0$ (рис. 4).

На рис. 5 показаны зависимости энергетической характеристики $R$ (13) сигнала ЗСП от расстройки частоты $\delta$ для различных моментов времени $\Delta>0$ с начала регистрации ЗСП при толщине ячейки $l=0.5 \lambda$ и $1.5 \lambda$. При возрастании $\Delta$ в этих спектральных зависимостях происходит возрастание контрастности и сужение внутридопплеровского резонанса с центром в точке $\delta=0$, что находится в соответствии с предыдущим рис. 4. Согласно рис. 6 , с повышением $\Delta$ амплитуда данного резонанса $A=R(\delta=0)$ уменьшается гораздо быстрее, чем его ширина $w$ (на полувысоте). Это обстоятельство необходимо учитывать при выборе в соответствующих экспериментах момента времени $\Delta$ для начала регистрации внутридопплеровских резонансов ЗСП (13) с оптимальными параметрами.
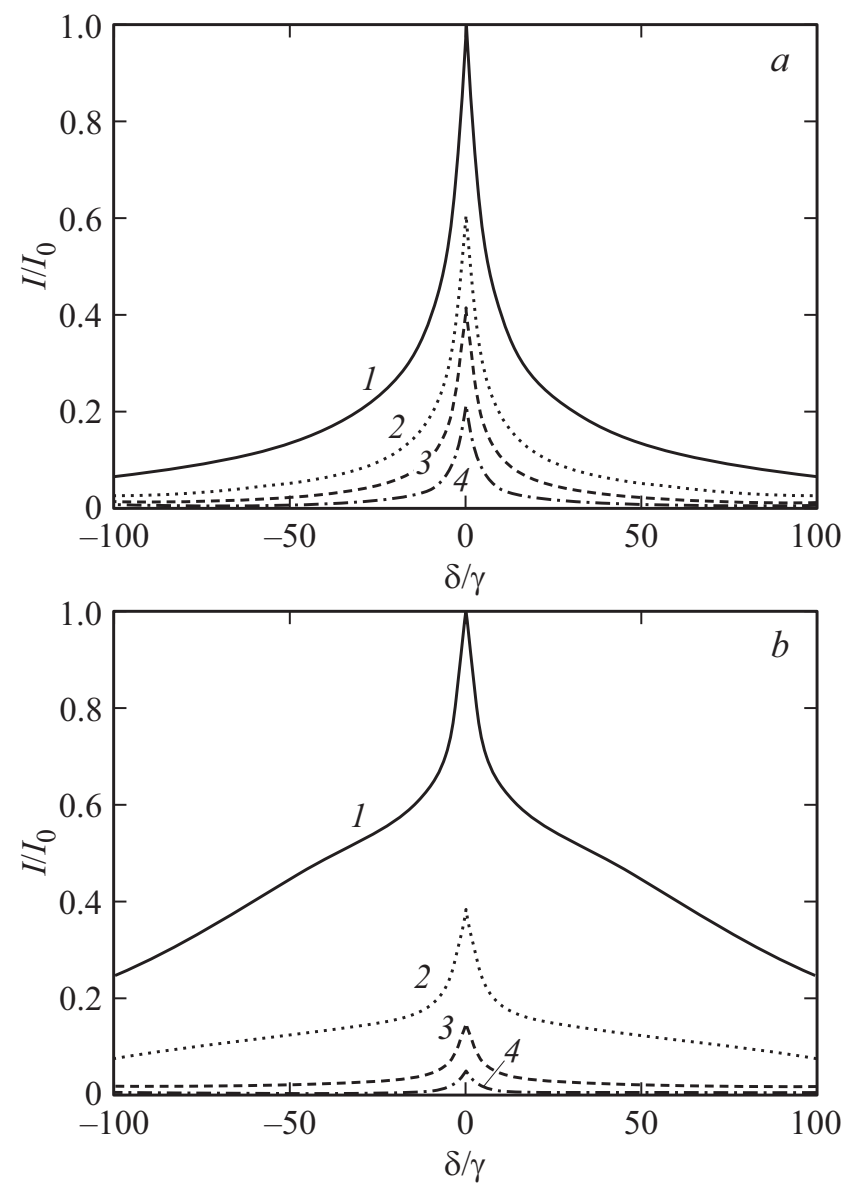

Рис. 4. Зависимость интенсивности $I$ сигнала ЗСП от расстройки частоты $\delta$ (в единицах $\gamma$ ) в моменты времени $t(k u)=0(1), 1(2), 2(3)$ и $5(4)$ при $l / \lambda=0.5(a)$ и $1.5(b)$, когда $k u=10^{2} \gamma \cdot I_{0}-$ значение интенсивности $I$ при $\delta=0$ в момент времени $t=t_{0}=0$. 

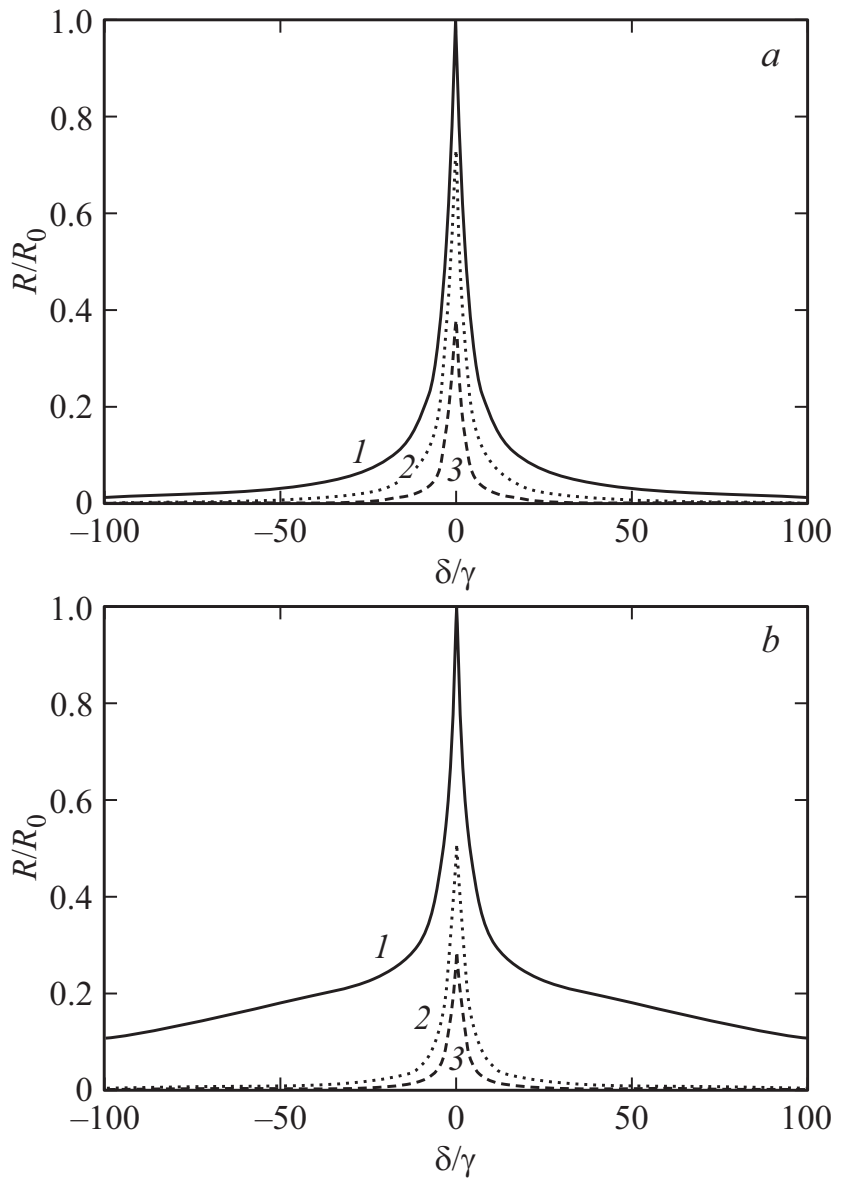

Рис. 5. Зависимость энергетической характеристики $R$ сигнала ЗСП от расстройки частоты $\delta$ (в единицах $\gamma$ ) при $\Delta(k u)=0(1), 2(2)$ и $10(3)$ для $l / \lambda=0.5(a)$ и $1.5(b)$, когда $k u=10^{2} \gamma \cdot R_{0}-$ значение $R$ при $\delta=0$ и $\Delta=0$.

На рис. 7 представлены зависимости амплитуды $A=R(\delta=0)$ и ширины $w$ энергетического спектра сигнала ЗСП (13) от толщины ячейки $l$ при регистрации этого сигнала, начиная с моментов времени $\Delta=5(k u)^{-1}$ и $10(k u)^{-1}$. Видно, что при целых значениях отношения $l / \lambda$ соответствующая амплитуда сигнала достигает минимума, а ширина - максимума. При полуцелых значениях $l / \lambda$ наблюдается обратная ситуация, когда при минимальной ширине $w$ имеет место максимум сигнала ЗСП. Действительно, согласно рассмотренным выше зависимостям на рис. 2 и 3, при полуцелых отношениях $l / \lambda$ возникает более продолжительный сигнал ЗСП с узким высококонтрастным резонансом в точке $\delta=0$ по сравнению со случаем целых значений $l / \lambda$. Эти особенности ярко проявляются в характеристиках интегральной по времени энергетической характеристики ЗСП (13) на рис. 7. Заметим, что радикальные различия между данными характеристиками ЗСП при целых и полуцелых отношениях $l / \lambda$ на рис. 7 постепенно уменьшаются с ростом толщины ячейки $l$ в области $l \gg \lambda$.

\section{Заключение}

Исследованные в настоящей работе сигналы ЗСП индуцируются линейной оптической поляризацией, которая предварительно создается возбуждающим когерентным излучением в ультратонком газовом слое ячейки. Такой сигнал ЗСП возникает уже в отсутствие исходного возбуждающего света (рис. 1). Поэтому рассмотренные нами резонансы ЗСП могут быть зарегистрированы и проанализированы с гораздо более высокой точностью и чувствительностью, чем упомянутые линейнооптические внутридопплеровские резонансы поглощения в ультратонких газовых ячейках [2-4]. Структура исследованных в настоящей работе резонансов и динамика ЗСП существенно зависят от отношения внутренней толщины ячейки $l$ к длине волны $\lambda$ лазерного излучения. Наиболее узкие и высококонтрастные внутридопплеровские резонансы ЗСП возникают, когда отношение $l / \lambda$ является полуцелым числом (особенно 0.5 или 1.5). Детектирование таких резонансов целесообразно осуществлять в спектре энергетической характе-
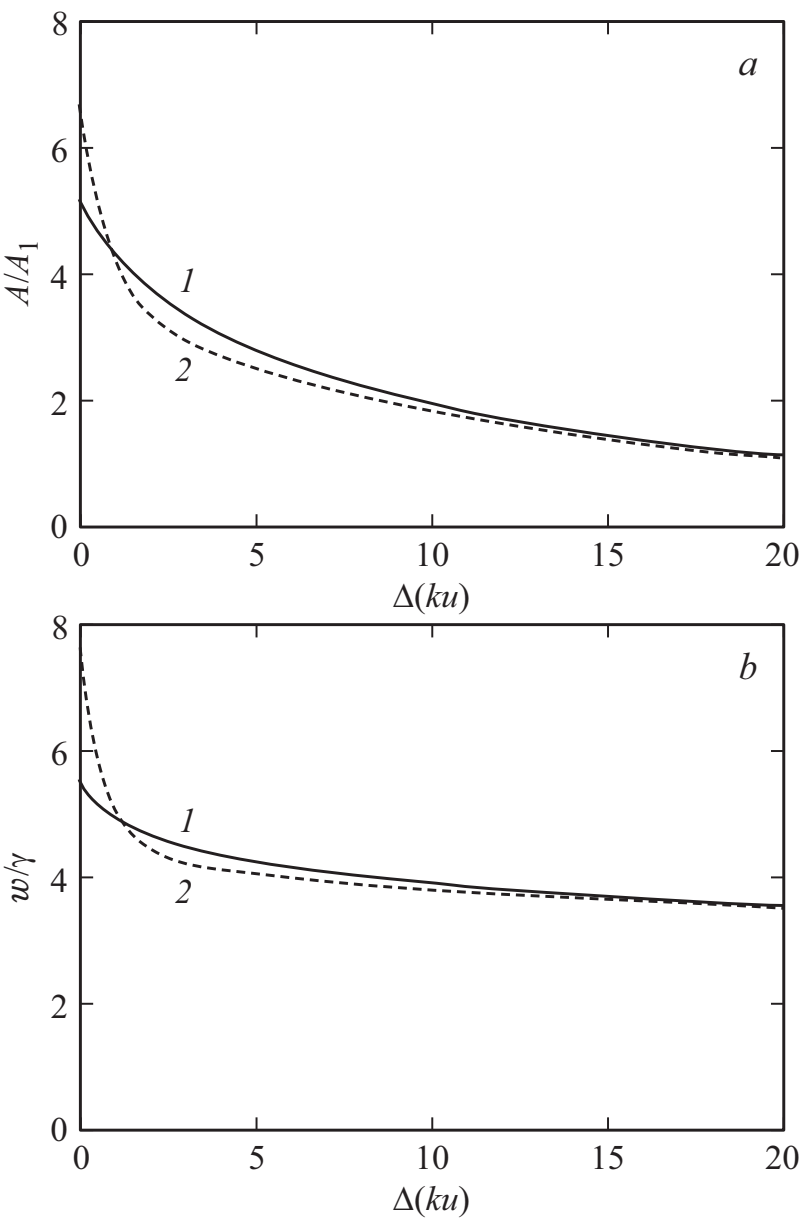

Рис. 6. Зависимость амплитуды $A(a)$ и ширины $w$ (в единицах $\gamma)(b)$ энергетической характеристики $R$ сигнала ЗСП от момента времени $\Delta>0$ (в единицах $\left.k^{-1} u^{-1}\right)$ при $l / \lambda=0.5(1)$ и $1.5(2)$, когда $k u=10^{2} \gamma$. Величина $A_{1}$ на рис. $6, a-$ значение амплитуды $A$ при $l=\lambda, \delta=0$ и $\Delta=0$. 

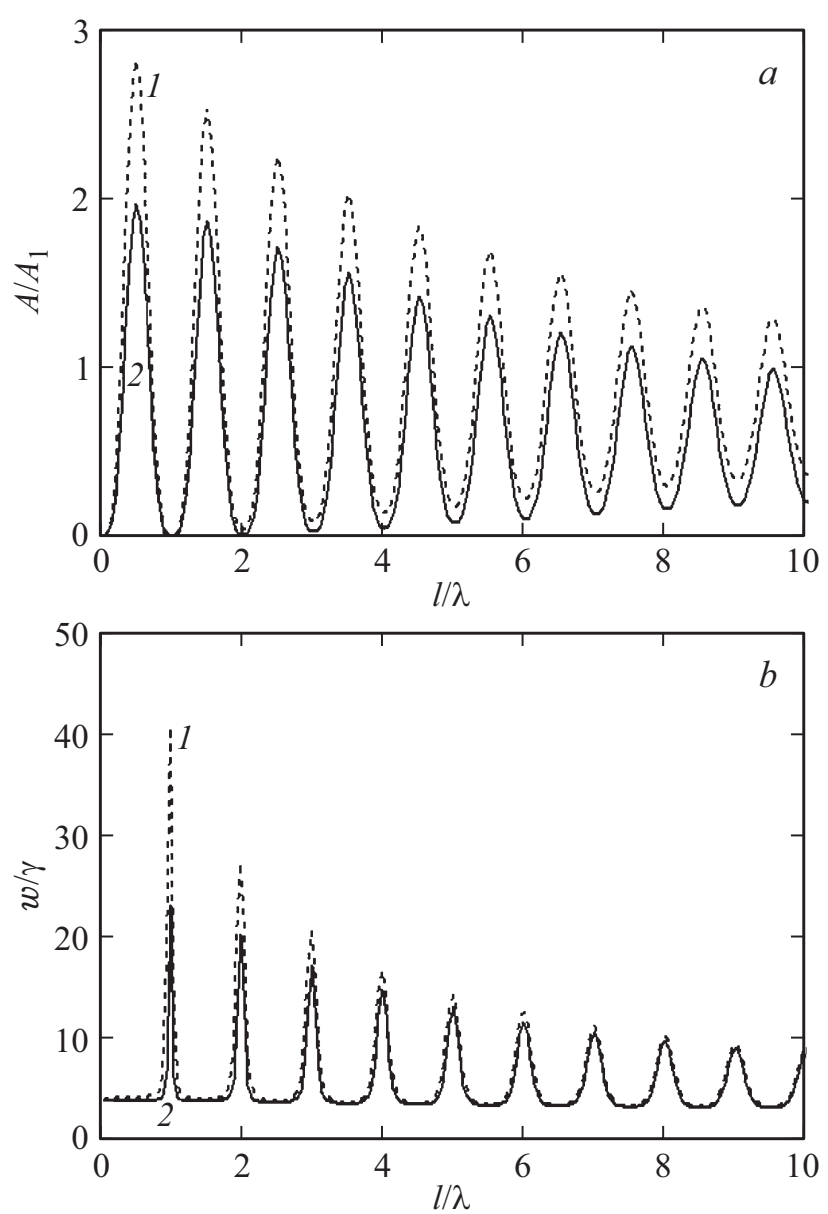

Рис. 7. Зависимости амплитуды $A(a)$ и ширины $w$ (в единицах $\gamma)(b)$ энергетической характеристики $R$ сигнала ЗСП от толщины ячейки $l$ (в единицах $\lambda$ ) при $\Delta(k u)=5(1)$ и $10(2)$, когда $k u=10^{2} \gamma$. Величина $A_{1}$ на рис. 7, $a-$ значение амплитуды $A$ при $l=\lambda, \delta=0$ и $\Delta=0$.

ристики ЗСП $R$ (13), начиная с некоторого момента времени $\Delta$ после прерывания исходного возбуждающего излучения. Установленные и проанализированные в настоящей работе внутридопплеровские резонансы ЗСП при определенных условиях могут найти применение в атомной (молекулярной) спектроскопии сверхвысокого разрешения [9], а также в качестве реперов для компактных стандартов частоты [10].

\section{Список литературы}

[1] Peyrot T., Sortais Y.R.P., Greffet J.-J., Browaeys A., Sargsyan A., Keaveney J., Hughes I.G., Adams C.S. // Phys. Rev. Lett. 2019. V. 122. N 11. P. 113401.

[2] Dutier G., Yarovitski A., Saltiel S., Papoyan A., Sarkisan D., Bloch D., Ducloy M. // Europhys. Lett. 2003. V. 63. N 1. P. 35.

[3] Vartanyan T.A., Lin D.L. // Phys. Rev. A. 1995. V. 51. N 3. P. 1959.

[4] Zambon B., Nienhuis G. // Opt. Commun. 1997. V. 143. N 4 6. P. 308.
[5] Bloch D., Ducloy M. // Advances in Atomic, Molecular, and Optical Physics. 2005. V. 50. P. 91.

[6] Laser and Coherence Spectroscopy / Ed. by Steinfeld J.S. NY:: Plenum Press, 1978. 529 р.; Лазерная и когерентная спектроскопия / Под ред. Стейнфелда Дж. М.: Мир, 1982. $629 \mathrm{c}$.

[7] Измайлов А.Ч. // Опт. и спектр. 2020. Т. 128. В. 8. С. 1070; Izmailov A.Ch. // Opt. Spectrosc. 2020. V. 128. N 8. P. 1074.

[8] Berman P.R., Malinovsky V.S. Principles of Laser Spectroscopy and Quantum Optics. Princeton University Press, 2011. $519 \mathrm{p}$.

[9] Demtroder W. Laser Spectroscopy: Basic Concepts and Instrumentation. Springer-Verlag, 1996. 924 p.

[10] Riehle F. Frequency Standards: Basics and Applications. WILEY-VCH, 2006. 525 p.; Риле Ф. Стандарты частоты: принципы и приложения. М.: Физматлит, 2009. 512 с. 